\title{
Clinical Presentation and Surgical Management of Abdominal Tuberculosis at M.Y. Hospital, Indore
}

\author{
Prabhat Shukla ${ }^{1}$, Pradeep Kumar Kothiya ${ }^{2}$, Faisal Ansari³ ${ }^{3}$, Abhineet Kumar Jain ${ }^{4}$ \\ ${ }^{1}$ Department of General Surgery, NSCB Medical College, Jabalpur, Madhya Pradesh, India. ${ }^{2}$ Department of General \\ Surgery, NSCB Medical College, Jabalpur, Madhya Pradesh, India. ${ }^{3}$ Department of General Surgery, Atal Bihari \\ Vajpayee Medical College, Vidisha, Madhya Pradesh, India. ${ }^{4}$ Department of General Surgery, Jabalpur Hospital and \\ Research Centre, Jabalpur, Madhya Pradesh, India.
}

\section{ABSTRACT}

\section{BACKGROUND}

Abdominal tuberculosis has been a common disease that poses challenge for the diagnosis of disease, as vague symptoms of the disease always lead to delay in diagnosis. This disease can be called as an imitator of other abdominal pathologies. A high index of doubt is necessary to make early and accurate diagnosis. Abdominal involvement can occur in body at various places like gastrointestinal tract, solid viscera, peritoneum and lymph nodes. Numerous investigations have to be used to help in making the accurate diagnosis of abdominal tuberculosis. Accurate diagnosis in early pathogenic stage and starting of antitubercular therapy and surgical treatment are important to prevent complications leading to morbidity and mortality of patients. Patients having long term symptoms are often weak debilitated, anaemic, malnourished, chronically ill and underweight which may be due to cachexia.

\section{METHODS}

This is a prospective observational study conducted from March 2016 to March 2018, in Mahatma Gandhi Medical College Associated M.Y. Hospital, among 47 patients with a diagnosis of abdominal tuberculosis confirmed histopathologically.

\section{RESULTS}

Maximum patients in our study belonged to 20 to 40 yrs. age group. 27\% were males and $72 \%$ were females. $8 \%$ patients were treated conservatively and $91 \%$ patients were offered operative treatment. Majority of patients presented with symptoms of abdominal pain (85\%), abdominal distension (85\%), vomiting (74\%), weight loss (74\%), fever (17\%), alteration in bowel habits $(21 \%)$ and lump in abdomen $(6 \%)$. Most common findings were of primary perforations (68\%), adhesions (46\%), plastered abdomen (34\%), ileocecal mass and ileocecal junction perforation (6\%). Ileal and jejunal strictures were minor findings. In majority of patients, ileostomy $(83 \%)$ was performed as life saving measure, adhesiolysis was done in 68\%, primary closure of perforation $(25 \%)$, resection and anastomosis $(8.5 \%)$.

\section{CONCLUSIONS}

Since the clinical presentations of abdominal tuberculosis are very non-specific and vague and the diagnostic criteria are limited, diagnosis has to be supported by additional tests and by retrospective analysis with reference to clinical patterns and underlying diseases. We also emphasize the importance of histopathological examination in establishing the diagnosis in poor resource settings.

\section{KEY WORDS}

Abdominal Tuberculosis, Ileocecal Mass, Perforation, Morbidity, Mortality
Corresponding Author: Pradeep Kumar Kothiya, C/o. RKJain, Maharana Pratap Ward, Navnivesh Colony, Near Bhagwati School, Garha, Jabalpur-4820003, Madhya Pradesh, India. E-mail: pradeepkothiya48@gmail.com

DOI: $10.14260 /$ jemds/2019/803

Financial or Other Competing Interests: None.

How to Cite This Article:

Shukla P, Kothiya PK, Ansari F, et al. Clinical presentation and surgical management of abdominal tuberculosis at my hospital, Indore. J. Evolution Med. Dent. Sci. 2019;8(49):3711-3714, $10.14260 /$ jemds $/ 2019 / 803$

Submission 13-05-2019,

Peer Review 22-11-2019,

Acceptance 29-11-2019,

Published 09-12-2019. 


\section{BACKGROUND}

Abdominal tuberculosis has been a common disease that poses a challenge for diagnosis of disease, as vague symptoms of the disease always lead to delay in diagnosis and result in problems. This disease can be called as a imitator of other abdominal pathology. A high index of doubt should be kept to make early and accurate diagnosis. Abdominal involvement can occur in body at various places like gastrointestinal tract, solid viscera peritoneum, lymph nodes. Numerous investigations have to be used to help in making the accurate diagnosis of abdominal tuberculosis. Accurate diagnosis in early pathogenic stage and starting of antitubercular therapy and surgical treatment are important to prevent complications leading to morbidity and mortality of patients.

Patients having long term symptoms are often weak debilitated, anaemic, malnourished, chronically ill, underweight may be cachexia. The abdomen on palpation may be normal or distended, doughy feel, and tenderness may be present in the right lower quadrant, GUARDING may be present. Peristalsis may be seen, and loops of intestine may also be seen with naked eye. If piled up omentum is present it may be felt as a nonspecific mass in the epigastric region. An ileocaecal mass suggestive of ileocecal TB may be felt high in the right iliac fossa or in the right lumber region. Shifting dullness and fluid thrill indicate the presence of ascites, while affected colon feels diffusely thick and tender. Loculated ascites, mesenteric cyst, and mesenteric abscess present as cystic mass. Hepatomegaly may be present in some patients. On per Rectal examination, anal fistulae, fissures, or stricture may be present. A maximum number of patients have very good response to standard antitubercular therapy and surgery plays role in some cases. Imaging investigation have a crucial and significant role in early and accurate diagnosis of abdominal tuberculosis because early diagnosis and accurate management of this condition is important. The clinical symptoms of abdominal tuberculosis are vague which is particularly right in case of peritoneal tuberculosis. When the intestine is involved the patient may present with gastrointestinal symptoms. Abdominal tuberculosis is mostly present in patients between the 30 and 50 yrs. of ages. Females are more than males by $2: 1$.

Tuberculosis (TB) is a life endangering pathology which can significantly affect any organ of the body and spread from one system to other system of the body.[1] Abdominal TB comprises around 5 percent of all cases of TB.[5] The prevalence of TB has significantly increased in both immunocompromised and immunocompetent. The primary site of TB is usually lung, after which it may spread to other organ systems of the body. The other routes of spread can be continuous involvement from adjacent tubercular lymph nodes or first involvement of extrapulmonary organ. The diagnosis of extrapulmonary TB is sometimes very difficult as it presents with vague clinical symptoms and radiological features and need high suspicion for identification of this pathology. The abdominal $\mathrm{TB}$, which is less prevalent as compared to pulmonary TB, can lead to complications which may lead to morbidity and mortality because it is diagnosed late due to its vague clinical features. ${ }^{[2]}$ Approximately $15 \%$ $25 \%$ of cases with abdominal TB have coexisting pulmonary TB. ${ }^{[3,4]}$ Hence, it becomes very important to recognize this disease with high level of suspicion more so in endemic areas.
Abdominal TB is a type of TB that affects the Gastrointestinal tract, Peritoneum, Abdominal lymph nodes and less affected are the solid organs in the abdomen (liver, pancreas, spleen). Abdominal tuberculosis may be due to a primary infection or the result of reactivation of a silent focus (post primary tuberculosis). The latter can happen due to immunocompromised states in patients presenting with severe infections, HIV, malnutrition etc.

With the rising numbers of HIV patients, there is also an increase in extra-pulmonary tuberculosis, that is, tuberculosis which Happens outside the lungs. One of the common types of extra-pulmonary tuberculosis is abdominal TB. Mycobacterium tuberculosis and Mycobacterium bovis are the Common causative organisms of this pathology. Spread of this disease occurs via blood, direct contact with primary focus or swallowing of sputum containing bacilli from the active pulmonary focus.

\section{Types of Abdominal Tuberculosis}

1. Ascitic: This kind of abdominal tuberculosis leads to large collections of fluid in the abdomen and with formation of nodules of 1-2 $\mathrm{mm}$ in size formed over the peritoneum (the inner lining of the abdomen) which are raised. It is also commonly called as wet type of abdominal tuberculosis.

2. Obstructive: This kind is also called dry type and leading to formation of adhesions over the omentum and loops of intestines which may later on become thick and rolled up leading to intestinal obstruction.

3. Glandular: This kind affects mesenteric lymph nodes which increase in size and become firm to hard in consistency and are less mobile. They can at times be felt through the abdominal wall. Some amount of ascites and intestinal obstruction may be present, but it is not characteristic.

Abdominal TB mounts to severe illness in children and in adults and may cause complications as bowel rupture can lead to death. The symptoms of chronic abdominal tuberculosis are slow, and include fever, and are usually present in two-thirds of patients, night sweats, malaise, weakness, anorexia, and weight loss. Abdominal pain is often vague and dull. When abdominal pain becomes colicky and severe is normally due to increased peristalsis to overcome obstruction, suggests intestinal obstruction. The pain is more often present in the right lower quadrant of abdomen and is often aggravated by eating food. Loose motions and occasionally vomiting tend to relieve the discomfort, which recurs later. It leads to watery and foul-smelling stools. Other features usually include flatulence, nausea, altered bowel habits, and borborygmi. Abdominal distension, if present, may be due to ascites or intestinal obstruction. Features which resemble duodenal ulcer may occur when the duodenum is affected. Bleeding from the rectum or haematemesis is rare. Gastrointestinal haemorrhage is occasionally severe and may be lifethreatening. Patients with intestinal involvement may also present with acute symptoms due to intestinal obstruction, perforation, or gangrene. Risk factors for development of abdominal TB include, HIV infection, diabetes mellitus, underlying malignancy, treatment with anti-tumour necrosis factor (TNF) agents, and use of peritoneal dialysis, cirrhosis.[610] 


\section{Diagnosis and Treatment of Abdominal Tuberculosis}

Diagnosis of abdominal tuberculosis is often clinical, but is confirmed with tests (blood test and other investigations). Treatment is with antitubercular drugs and surgery in combination or alone depending upon individual patient requirement. Diagnosis of abdominal tuberculosis can be made with the help of Mantoux test, liver function tests, ESR, serum albumin levels, x-rays/ ultrasound/ CT scan abdomen, endoscopy, colonoscopy and laparoscopy. Fine needle aspiration cytology, peritoneal biopsy, ascitic fluid assessment with PCR assay and Quanti Feron - TB test is all effective in reaching a final accurate diagnosis. Treatment is with antitubercular drugs and/or surgery. Antitubercular drugs are given for at least six months initially; depending upon the results of response to therapy, treatment is continued further (12- 18 months). Common drugs employed are ethambutol, pyrazinamide, isoniazid and rifampicin. Surgical treatment is usually conservative. Correction for strictures with stricturoplasty and perforations in the intestines with resection and anastomosis is recommended. Intestinal obstruction is initially treated with drug therapy before proceeding to corrective surgeries.

We wanted to study the incidence, clinical presentation, management (conservative or surgical) and its outcome, morbidity and mortality in patients of abdominal tuberculosis.

\section{METHODS}

It is a prospective observational study including 47 patients were part of this study conducted from March 2016 to March 2018 with a diagnosis of abdominal tuberculosis confirmed histopathologically carried out in Mahatma Gandhi Medical college associated M.Y. Hospital. Sample size was taken based on the convenience of the study. All the cases diagnosed as abdominal tuberculosis, including gastrointestinal tract, mesenteric lymph nodes, peritoneum, or other intraabdominal solid organs were retrieved. The detailed patient information in form of age, sex, residential address, symptoms, clinical details, laboratory results and treatment outcome were recorded. All specimens had undergone Ziehl-Neelsen (ZN) staining to identify the tubercular bacilli.

The diagnosis of abdominal tuberculosis was made in all the cases fulfilling following criteria(11)

- In ascitic/biopsy specimens, acid fast stain positivity for M. tuberculosis was made.

- On histopathological examination of excised/ resected specimens from abdomen Identification of caseating granulomas was done.

- $\quad$ Response of the patient to anti-tubercular treatment was present.

HIV (Human Immunodeficiency Virus) screening was done in all the cases and cases with positive HIV status were excluded from the study.

\section{Statistical Analysis}

The statistical package for social science $\{$ SPSS $\}$ version 20 will be used for data analysis. Mean, median, and SD are used to describe quantitative data. Qualitative data are summarized using frequency and percentage.

\section{RESULTS}

\begin{tabular}{|c|c|}
\hline Age & No. of Patients \\
\hline $10-20$ yrs. & 2 \\
\hline $20-40$ yrs. & 44 \\
\hline$>40$ Yrs. & 1 \\
\hline \multicolumn{2}{|c|}{ Table 1. Age Distribution } \\
\hline
\end{tabular}

\begin{tabular}{|c|c|c|}
\hline Sex & No. of Patients & Percentage \\
\hline Females & 13 & 27.6 \\
\hline Males & 34 & 72.34 \\
\hline \multicolumn{3}{|c|}{ Table 2. Sex Distribution } \\
\hline
\end{tabular}

\begin{tabular}{|c|c|c|}
\hline Management & No of Patients & Percentage \\
\hline Operative & 43 & 91.4 \\
\hline Conservative & 4 & 8.5 \\
\hline \multicolumn{3}{|c|}{ Table 3. Decision of Treatment } \\
\hline
\end{tabular}

\begin{tabular}{|c|c|c|}
\hline Clinical Feature & No. of Patients & Percentage (\%) \\
\hline Abdominal Distension & 40 & 85 \\
\hline Fever & 17 & 36 \\
\hline Vomiting & 35 & 74 \\
\hline Alteration in bowel habits & 10 & 21 \\
\hline Weight loss & 35 & 74 \\
\hline Abdominal Pain & 40 & 85 \\
\hline Lump in abdomen & 3 & 6 \\
\hline \multicolumn{2}{|c|}{ Table 4. Clinical Features } \\
\hline
\end{tabular}

\begin{tabular}{|c|c|c|}
\hline Site of Lesion & No. of Patients & Percentage \\
\hline Ileal Stricture & 1 & 2 \\
\hline Ileocecal Mass & 3 & 6.3 \\
\hline Ileocecal Junction Perforation & 3 & 6.3 \\
\hline Adhesions & 22 & 46 \\
\hline Plastered Abdomen & 16 & 34 \\
\hline Primary Perforations & 32 & 68 \\
\hline Jejunal Strictures & 1 & 2 \\
\hline \multicolumn{2}{|c|}{ Table 5. Site of Lesion / Intraoperative Finding. } \\
\hline
\end{tabular}

\begin{tabular}{|c|c|c|}
\hline Management & No. of Patients & Percentage \\
\hline Resection Anastomosis & 4 & 8.5 \\
\hline Right hemicolectomy & 3 & 6.3 \\
\hline Stricturoplasty & 2 & 4.2 \\
\hline Ileostomy & 39 & 82.97 \\
\hline Adhesiolysis & 32 & 68 \\
\hline Primary Closure of Perforation & 12 & 25 \\
\hline \multicolumn{2}{|c|}{ Table 6. Surgical Management } \\
\hline
\end{tabular}

\section{DISCUSSION}

The total number of patients in our study group was 47 , with males and females being $34(72.34 \%)$ and 13 (27.6\%), respectively. Charokar $\mathrm{K}$ reported $61 \%$ males and $39 \%$ females in their study group.(1) The youngest and the oldest patient in our study group were 16 years old boy and a 67 years old lady, respectively. The mean age of the patients in our study was 26.4 years. Sadia J $^{(2)}$ reported mean age in their study to be 35.9 years. This difference in mean ages in this study and hers is probably due to the larger sample size of 161 patients in her study as compared to our sample size of 47 patients. Majority of study patients $85 \%$ were in the undernourished category and $15 \%$ were in the normal weight category as per the WHO-BMI classification. This points to a strong association between malnourishment and tuberculosis. Tuberculosis affects primarily the malnourished and later on due to anorexia and malabsorption leads to further malnourishment. Similar findings have been reported by other researchers like Charokar $\mathrm{K},(1)$ who reported $68 \%$ of his study group patients to be in underweight category and $32 \%$ in the normal weight category.(1) Thirty five of our patients presented to our surgery emergency with features suggestive 
of peritonitis and Twelve patients with acute intestinal obstruction and sub-acute intestinal obstruction. The most frequent complaint in history was pain $(85 \%)$ followed by vomiting (74.4\%), weight loss (74\%), fever (36\%) and altered bowel habits $(21 \%)$. Sharma MP(3) have reported similar findings in their research. Study found that primary perforation was the commonest intra-operative finding be it Ileal or jejunal perforation followed in decreasing order of frequency by multiple small bowel perforations, ileo-cecal mass, adhesions or bands, plastered abdomen, single or multiple small bowel strictures, small bowel strictures with impending perforation. Charokar K and Sadia J have reported similar results in their research.(1,2) Ileostomy was the commonest surgical intervention performed in our study in 39 Patients (83\%) followed by adhesiolysis (68\%), primary repair of the perforated viscus (25\%), right hemi-colectomy or limited ileocecal resection, stricturoplasty and in a solitary case only omental and peritoneal biopsy with peritoneal lavage was done. Different authors and researchers, Charokar $\mathrm{K}$, Sadia J and Ali $\mathrm{N}^{(4)}$ have suggested and reported a multitude of surgical procedures in the surgical management of abdominal tuberculosis but it has been rightly stated by Sabooni K.(5) that given the diverse presentation and characteristics of abdominal tuberculosis, no definite surgical procedure can be regarded as the standard of care. Hence, we may say that the appropriate surgical procedure and decision has to be taken by operating surgeon based on the performance status and general condition of the patient at the time of surgery, the site and extent of the disease and the expertise of the operating surgeon.

In our study group four patients were treated conservatively and had recovered without any surgical intervention. There was mortality of two patients was due to poor general condition, faecal peritonitis and ongoing septicaemia at the time of admission. Similar findings have been reported by Sadia J.(2)

\section{CONCLUSIONS}

Clinical presentations of abdominal tuberculosis are very nonspecific and vague, and the diagnostic criteria are limited.
Therefore, diagnosis has to be supported by additional tests and by retrospective analysis with reference to clinical patterns and underlying diseases. We also emphasize the importance of histopathological examination in establishing the diagnosis in poor resource settings.

\section{REFERENCES}

[1] Charokar K, Garg N, Jain AK. Surgical management of abdominal tuberculosis: a retrospective study from Central India. Int Surg J 2016;3(1):23-31.

[2] Sadia J, Mehmood N, Khan NM. Surgical management of acute presentation and outcome of patients with complicated abdominal tuberculosis. J Rawalpindi Med Coll (JRMC) 2016;20(2):108-12.

[3] Sharma MP, Bhatia V. Abdominal tuberculosis. Indian J Med Res 2004;120(4):305-15.

[4] Ali N, Hussain M, Israr M. Tuberculosis as a cause of small bowel obstruction in adults. Gomal J Med Sci 2011;9(2):233-5.

[5] Sabooni K, Khosravi MH, Pirmohammad H, et al. Tuberculosis peritonitis with features of acute abdomen in HIV infection. Int J Mycobacteriol 2015;4(2):151-3.

[6] Rosado E, Penha D, Paixao P, et al. Abdominal tuberculosis - imaging findings. Educational exhibit ECR. 2013: C-0549.

[7] Mukewar S, Mukewar S, Ravi R, et al. Colon tuberculosis: endoscopic features and prospective endoscopic followup after anti-tuberculosis treatment. Clin Transl Gastroenterol 2012;3(10):e24.

[8] Horvath KD, Whelan RL. Intestinal tuberculosis: return of an old disease. Am J Gastroenterol 1998;93(5):692-6.

[9] Akhan 0, Pringot J. Imaging of abdominal tuberculosis. Eur Radiol 2002;12(2):312-23.

[10] Sharma SK, Mohan A. Extrapulmonary tuberculosis. Indian J Med Res 2004;120(4):316-53.

[11] Debi U, Ravisankar V, Prasad KK, et al. Abdominal tuberculosis of the gastrointestinal tract: revisited. World J Gastroenterol 2014;20(40):14831-40. 